\title{
Heat Transfer in Enclosure of Composite Material with Fibers Arranged in Various Geometrical Arrays
}

\author{
Amina H. Dhaef \\ Faculty in Engineering college \\ University of Wasit \\ Mechanical Department
}

\begin{abstract}
This paper presents a numerical investigation for natural convection of air in a three dimensional inclined annulus enclosure. This study wills exam the effect of radius ratio of an annulus made from graphite/epoxy laminated composite material on heat transfer taking two types of optimization of effective thermal conductivity in consideration: minimization and maximization of thermal conductivity. The annulus enclosure is filled with porous media between two concentric cylinders with 12 fins attached to the inner cylinder. Two cases are taken for the inclination angle of the annulus: horizontal and vertical annulus. The system is under steady state condition and constant walls temperature boundary condition. The parameters affected on the system are modified Rayleigh number $\left(10 \leq \mathrm{Ra}^{*} \leq 500\right)$, the annulus inclination angle $\delta\left(0^{\circ}\right.$ and $\left.90^{\circ}\right)$ and the radius ratio $\mathrm{Rr}=(\mathrm{RI} / \mathrm{RO})=0.2,0.3$, 0.4 and 0.5 .
\end{abstract}

For all parameters, results showed that Nusselt number decrease with the decrease of the radius ratio $\mathrm{Rr}$ (which means larger gap) for the outer cold cylinder. the average $\mathrm{Nu}$ number increases with an increase in modified Rayleigh number and decrease with the increase of $\delta$ for high values of $\mathrm{Ra}^{*}$, but hardly affected by $\delta$ for low values of $\mathrm{Ra}^{*}$. The deviation between the average $\mathrm{Nu}$ for the maximization and minimization of the thermal conductivity is equal to $5.1 \%$ for horizontal annulus $\delta=0^{\circ}$ and $10 \%$ for vertical annulus $\delta=90^{\circ}$. Local $\mathrm{Nu}$ increases with the length of the cylinder and the effect of the fins attached to the inner cylinder is more significant for the horizontal cylinder because of its hindering effect. A correlation for the average Nusselt number in terms of $\mathrm{Ra}^{*}$ and $\delta$, has been developed for the outer cold cylinder.

\section{General Terms}

$\mathrm{Cp}$ : Specific heat at constant pressure $\left(\mathrm{kJ} / \mathrm{kg}{ }^{\circ} \mathrm{C}\right), \mathrm{g}$ : Acceleration due to gravity $\left(\mathrm{m} / \mathrm{s}^{2}\right), \mathrm{H}_{\mathrm{f}}$ : Fin length $(\mathrm{m})$ $\mathrm{k}_{\mathrm{f}}$ : Thermal conductivity of the fluid (W/m K), ks: Thermal conductivity of the solid $(\mathrm{W} / \mathrm{m} \mathrm{K}), \mathrm{k}_{\text {eff. }}$ : Effective thermal conductivity of the porous media (W/m K), K: Permeability $\left(\mathrm{m}^{2}\right)$, l: cylinder length $(\mathrm{m}), \mathrm{L}$ : Dimensionless cylinder length, $\mathrm{Nu}_{\text {Local } 1}$ : Local Nusselt number on the inner surface, $\mathrm{Nu}_{\text {Local } 2}$ : Local Nusselt number on the outer surface, $\mathrm{Nu}_{1}$ : Mean Nusselt number on the inner surface, $\mathrm{Nu}_{2}$ : Mean Nusselt number on the outer surface, p: Pressure (N/m2), q: Local heat flux (m), r: Radial coordinate (m), R: Dimensionless radial coordinate, $\mathrm{Ra}^{*}$ : Modified Rayleigh number, Rr: Radius ratio, S: fin pitch $(\mathrm{m}), \mathrm{T}$ : Temperature $(\mathrm{K})$, ur,u $\phi$, uz: velocity component in $\mathrm{r}, \phi$ and $\mathrm{z}$ - direction $(\mathrm{m} / \mathrm{s})$, Ur, U $\phi, \mathrm{Uz}$ : Dimensionless velocity component in $\mathrm{R}, \phi$ and $\mathrm{Z}$ direction, $\mathrm{x}$, y, z: Cartesian coordinate system (m), Z: Dimensionless axial coordinate, $\alpha_{\mathrm{f}}$ : Fluid thermal diffusivity $\left(\mathrm{m}^{2} / \mathrm{s}\right), \alpha_{\mathrm{s}}$ : Solid thermal diffusivity, $\alpha_{\text {eff. }}$ : Effective thermal diffusivity $\left(\mathrm{m}^{2} / \mathrm{s}\right)$, $\beta$ : Volumetric thermal expansion coefficient (1/K) $\theta$ : Dimensionless temperature, $\psi \mathrm{r}, \psi \phi, \psi \mathrm{z}$ : Vector potential component in R, $\phi$ and $\mathrm{Z}$ - direction, $\mu_{f}$ : Dynamic viscosity of fluid (Pa.s), FAI: angular direction.

\section{Keywords}

Natural convection, laminar flow, porous media, graphite/epoxy, composite material.

\section{INTRODUCTION}

The spacious advantages of the fiber reinforced multilayer composite materials like high strength-to-density ratio, stiffness-to-density ratio, high corrosion resistance and plasticity as compared with most materials cause these materials to be interested significantly in modern engineering applications. Most of these unique advantages are because of two properties of these materials i.e. the ability to change the fibers' orientations of every layer to meet the design requirements and combining different physical, mechanical and thermal properties of various materials.

Composite materials are used in various designs to improve the characteristic of various constructions and reduce their weight. Many researchers and engineers studied the properties of these materials and the problems of obtaining structural elements based upon them. Composite materials have been developed for a wide range of industrial applications, including electronic cooling, satellite, piping, pressure vessels, fluid reservoirs, aerospace components. Composite materials have many favorable properties, including high strength to- weight ratios and high corrosion resistances. The properties of these materials and the problems of obtaining structural elements based upon them have been studied by researchers and engineers all over the world [1]. Any property of a composite which is made of two (or more) materials has the value which is the resultant of a few factors. Obviously, the most important are the values of a certain property of each constituent material. However, one of the factor that also influences the resultant value of a property of a composite as a whole is its geometrical structure. Such resultant properties are commonly called effective properties of a composite. Temperature is the most important of all environmental factors affecting the behavior of composite materials, mainly because composites are rather sensitive to temperature and have relatively low effective thermal conductivity. For instance, advanced composites for engineering applications are characterized with low density providing high specific strength and stiffness, low thermal conductivity resulting in high heat insulation [2].

As a first time [3] presented an exact analytical solution for steady conductive heat transfer in multilayer spherical fiber reinforced composite laminates. The temperature distribution of laminate was obtained under the general boundary conditions that include different conditions which are conduction, convection, and radiation both inside and outside the sphere. The separation of variables method was used In order to obtain the exact solution, and the set of equations related to the coefficient of Fourier-Legendre series of temperature distribution was solved using the recursive Thomas algorithm. 
[4] As a thermal management system, a sandwich construction was developed to have both superior thermal conductivity and structural integrity. The sandwich construction consists of a carbon foam core and unidirectional graphite/epoxy composite face sheets. An emphasis was put on enhancing the thermal conductivity of each phase of sandwich construction as well as interface between the phases. A commerciallyavailable carbon foam was characterized mechanically and thermally. Property variation and anisotropy were observed with the highly conductive graphitic carbon foam. Co-curing of the composite face sheets with the carbon foam core was demonstrated to minimize the thickness of the adhesive layer between the face sheets and the core to produce the best construction of those tested. Comparison made with an adhesively bonded specimen shows that the co-curing is a more efficient method to enhance the through-thickness conductivity. Parametric studies with an analytic model indicate that degree of enhancement in the overall throughthickness conductivity of the sandwich construction from the enhancement of each component including the foam core, face sheet and the bonding methods.

[5] Developed an analytical model including the orientational distribution, aspect ratio, volume fraction, permeability of the fillers, and membrane non-idealities - to describe molecular transport in composite membranes with tubular fillers. Using the model, the effects of various structural and operational parameters of the composite membrane were assessed. It was found that the filler volume fraction, as opposed to the filler orientation, intrinsic permeability, and aspect ratio, had the most significant impact on the membrane permeability. Several case studies of binary mixture separations by ideal and non-ideal composite membranes with tubular fillers were discussed.

[6] Obtained an analytical solution for two-dimensional transient heat conduction in fiber-reinforced multilayer cylindrical composites. Separation of variables method is employed to develop the transient temperature fields. In the polar coordinates, the presented analytical solution contains trigonometric series and Bessel series. Both the sine series and the cosine series are included in the trigonometric series. To deal with the continuity conditions at the interfaces, the initial parameter method is used and the solution is derived only by operating two by two matrices. The solving procedure is performed directly in time domain and the Laplace transform is avoided. The effect of the fibers' angle on the transient heat conduction behaviors is investigated.

[7] Studies the thermal radiative transfer in silica aerogel and silica aerogel composite insulation materials (a xonotliteaerogel composite and a ceramic fiber-aerogel composite). The spectral transmittances of silica aerogel, xonotlite-type calcium silicate, and ceramic fiber insulation materials-all considered semi-transparent mediums capable of absorbing, emitting, and scattering thermal radiation-are measured with a Fourier transform infrared spectrometer (FTIR) at different infrared wavelengths ranging from 2.5 to $25 \mu \mathrm{m}$. The spectral transmittances are used to determine the specific spectral extinction coefficient and the specific Rosseland mean extinction coefficient of each sample. The radiative conductivity of each sample, deduced from the overall thermal conductivity measured using the transient hot-strip (THS) method, is compared against diffusion approximation predictions by using the measured spectral extinction coefficient. The results show that the spectral extinction coefficients of the samples are strongly dependent on the wavelength, particularly in the short wavelength regime (b10 $\mu \mathrm{m})$. The total Rosseland means extinction coefficients of the samples all decrease with increasing temperature. The radiative conductivities are almost proportional to the cube of temperature, decreasing as sample densities increase.

\section{OBJECTIVE OF THE RESEARCH}

In the present research the natural convection heat transfer is investigated in a horizontal and vertical annulus used as heat exchanger. The effect of the radius ratio was investigated. Two types of optimization will be performed in terms of effective thermal conductivity: minimization and maximization of thermal conductivity. Graphite/epoxy composite material is used for the annulus based on previous experimental work. The ranges of the parameters affected on the study are modified Rayleigh number $\left(10 \leq \mathrm{Ra}^{*} \leq 500\right)$ and the annulus inclination angle $\delta(0$ and 90$)$ and the radius ratio $\mathrm{Rr}=0.2,0.3,0.4$ and 0.4 .

\section{MATHEMATICAL MODEL}

The schematic drawing of the geometry and the Cartesian coordinate system employed in solving the problem is shown in Fig.1.
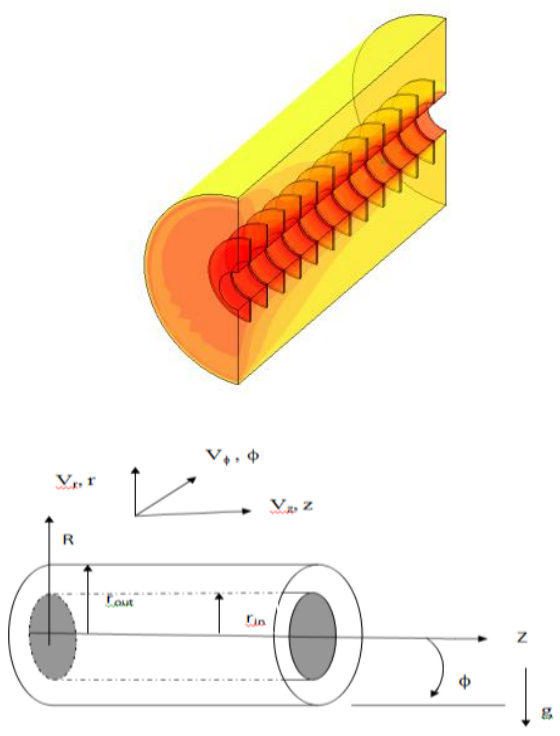

Fig. 1 Geometry and coordinates system

In order to model the incompressible flow in the porous medium, the steady-state equations of the Darcy flow model, namely, the mass, the momentum (Darcy), the energy conservation laws and the Boussinesq's approximation are employed. These equations in vectorial notation are given by [8].

\section{GOVERNING EQUATIONS}

The conservation equations of mass, momentum and energy in steady state and the supplementary equation are:

$\rho=\rho_{2}\left\{1-\beta\left(T-T_{2}\right)\right\}$

Where:

$\beta=\frac{1}{\rho} \frac{\partial \rho}{\partial T}$

$\beta$ Is the thermal coefficient of the volume expansion, this constant is evaluated at $T_{2}$ which is the temperature at the inner surface of the outer cylinder, $\rho_{2}$ is the density at $T_{2}$ and $\rho$ is the density at T, [9]. This technique is called Boussinesq's approximation. 


\subsection{Mass Conservation}

$\frac{\partial u_{r}}{\partial r}+\frac{u_{r}}{r}+\frac{1}{r} \frac{\partial u_{\emptyset}}{\partial \emptyset}+\frac{\partial u_{z}}{\partial z}$

\subsection{Momentum Equations}

The most common model used for flow in the porous media is the Darcy flow model. Darcy's law states that the volume average velocity through the porous material is proportional with the pressure gradient. In three dimensional flows, the Darcy's model [10] is:

\subsubsection{Momentum Equation in Radial Direction}

$u_{r}=\frac{K}{\mu_{f}}\left[-\frac{\partial p}{\partial r}-\rho g \cos \emptyset \cos \delta\right]$

\subsubsection{Momentum Equation in Angular Direction}

$u_{\emptyset}=\frac{K}{\mu_{f}}\left[-\frac{\partial p}{\partial \emptyset}+\rho g \sin \emptyset \cos \delta\right]$

\subsubsection{Momentum Equation in Axial Direction}

$u_{z}=\frac{K}{\mu_{f}}\left[-\frac{\partial p}{\partial z}-\rho g \sin \delta\right]$

\subsection{Energy Equation}

$\frac{\partial\left(\rho C_{p} T\right)}{\partial t}+u_{r} \frac{\partial\left(\rho C_{p} T\right)}{\partial r}+\frac{u_{\emptyset}}{r} \frac{\partial\left(\rho C_{p} T\right)}{\partial \emptyset}$

$+u_{z} \frac{\partial\left(\rho C_{p} T\right)}{\partial z}=\frac{1}{r} \frac{\partial}{\partial r}\left\{r \frac{\partial(k T)}{\partial r}\right\}+\frac{1}{r^{2}} \frac{\partial^{2}(k T)}{\partial \emptyset^{2}}$

$+\frac{\partial^{2}(k T)}{\partial z^{2}}+\mu \Phi$

Where $\Phi$ is viscous dissipation function.

\subsection{Fin Equation}

Within the fin itself, the energy equation is [11]:

$\frac{\partial T}{\partial r}+\frac{T}{r}+\frac{1}{r} \frac{\partial T}{\partial \theta}+\frac{\partial T}{\partial z}=0$

A vorticity vector $\Omega$ and a vector potential $\Psi$ with its components are [11]:

$\Psi=\left(\psi_{r}, \psi_{\phi}, \psi_{z}\right)$

Defined by:

$U=\alpha_{e f f} \nabla \mathrm{X} \Psi$

$\nabla^{2} \psi_{r}=\frac{1}{R} \frac{\partial U_{Z}}{\partial \emptyset}-\frac{\partial U_{\emptyset}}{\partial Z}$

$\nabla^{2} \psi_{\varnothing}=\frac{\partial U_{r}}{\partial Z}-\frac{\partial U_{z}}{\partial R}$

$\nabla^{2} \psi_{z}=\frac{1}{R} \frac{\partial\left(R U_{\emptyset}\right)}{\partial R}-\frac{1}{R} \frac{\partial U_{r}}{\partial \emptyset}$

\section{NON DIMENSIONAL VARIABLES}

The characteristic length for the present study is $r_{2}$ [9] to convert the governing equations to the dimensionless form, the dimensionless magnitudes must be defined as follow:

$$
\begin{gathered}
R=\frac{r}{r_{2}}, Z=\frac{z}{r_{2}}, U_{r}=\frac{u_{r} l}{\alpha_{e f f} .}, U_{\emptyset}=\frac{u_{\emptyset} l}{\alpha_{e f f .}}, U_{z}=\frac{u_{z} l}{\alpha_{e f f} .} \\
\theta=\frac{\left(T-T_{2}\right)}{\left(T_{1}-T_{2}\right)}, P=\frac{p K l}{\alpha_{e f f .} \mu_{f} r_{2}}, R_{a}^{*}=\frac{g \beta K\left(T_{1}-T_{2}\right)\left(r_{2}-r_{1}\right)}{\alpha_{e f f .} \vartheta} \\
S_{1}=\frac{s}{2 r_{2}}, S_{2}=\frac{\frac{s}{2}+t}{r_{2}}, H_{1}=\frac{H_{f}}{r_{2}}
\end{gathered}
$$

International Journal of Computer Applications (0975 - 8887)

Volume 125 - No.8, September 2015

Substitute these dimensionless magnitudes in the governing equations. Alternative expressions of equation (4.3) may be written in terms of $\psi_{r}, \psi_{\emptyset}, \psi_{z}$ as:

$$
\begin{aligned}
U_{r} & =\left(\frac{1}{R} \frac{\partial \psi_{z}}{\partial \emptyset}-\frac{\partial \psi_{\emptyset}}{\partial Z}\right) \\
U_{\emptyset} & =\left(\frac{\partial \psi_{r}}{\partial Z}-\frac{\partial \psi_{z}}{\partial R}\right) \\
U_{z} & =\frac{1}{R}\left(\frac{\partial\left(R \psi_{\varnothing}\right)}{\partial R}-\frac{\partial \psi_{r}}{\partial \emptyset}\right)
\end{aligned}
$$

Taking curl of momentum equations to eliminate pressure terms, the momentum equations will be:

$$
\begin{aligned}
& R_{a}^{*} \frac{l}{\left(r_{2}-r_{1}\right)}\left(\frac{1}{R} \sin \delta \frac{\partial \theta}{\partial \emptyset}+\sin \emptyset \emptyset \cos \delta \frac{\partial \theta}{\partial Z}\right)=-\frac{\partial^{2} \psi_{r}}{\partial R^{2}}- \\
& \frac{1}{R^{2}} \frac{\partial\left(R \psi_{r}\right)}{\partial R}-\frac{2}{R} \frac{\partial \psi_{r}}{\partial R}-\frac{1}{R^{2}} \frac{\partial^{2} \psi_{r}}{\partial \emptyset^{2}}-\frac{\partial^{2} \psi_{r}}{\partial Z^{2}}-\frac{2}{R} \frac{\partial \psi_{z}}{\partial Z} \\
& R_{a}^{*} \frac{l}{\left(r_{2}-r_{1}\right)}\left(\cos \emptyset \cos \delta \frac{\partial \theta}{\partial Z}-\sin \delta \frac{\partial \theta}{\partial R}\right)=-\frac{\partial^{2} \psi_{\emptyset}}{\partial Z^{2}}-\frac{\partial^{2} \psi_{\emptyset}}{\partial R^{2}}- \\
& \frac{1}{R^{2}} \frac{\partial^{2} \psi_{\emptyset}}{\partial \emptyset^{2}}-\frac{2}{R^{2}} \frac{\partial \psi_{r}}{\partial \emptyset}+\frac{\psi_{\emptyset}}{R^{2}}-\frac{1}{R} \frac{\partial \psi_{\emptyset}}{\partial R}
\end{aligned}
$$

$R_{a}^{*} \frac{l}{\left(r_{2}-r_{1}\right)} \cos \delta\left(\frac{1}{R} \cos \emptyset \frac{\partial \theta}{\partial \emptyset}+\sin \emptyset \frac{\partial \theta}{\partial R}\right)=-\frac{\partial^{2} \psi_{z}}{\partial R^{2}}-\frac{1}{R} \frac{\partial \psi_{z}}{\partial R}-$

$\frac{1}{R^{2}} \frac{\partial^{2} \psi_{z}}{\partial \emptyset^{2}}-\frac{\partial^{2} \psi_{z}}{\partial Z^{2}}$

The energy equation is:

$\left(\frac{1}{R} \frac{\partial \psi_{z}}{\partial \emptyset}-\frac{\partial \psi_{\emptyset}}{\partial Z}\right) \frac{\partial \theta}{\partial R}+\frac{1}{R}\left(\frac{\partial \psi_{r}}{\partial Z}-\frac{\partial \psi_{z}}{\partial R}\right) \frac{\partial \theta}{\partial \emptyset}+\left(\frac{\psi_{\emptyset}}{R}+\frac{\partial \psi_{\emptyset}}{\partial R}-\right.$

$\left.\frac{1}{R} \frac{\partial \psi_{r}}{\partial \emptyset}\right) \frac{\partial \theta}{\partial Z}=\frac{l}{r_{1}}\left(\frac{\partial^{2} \theta}{\partial R^{2}}+\frac{1}{R} \frac{\partial \theta}{\partial R}+\frac{1}{R^{2}} \frac{\partial^{2} \theta}{\partial \emptyset^{2}}+\frac{\partial^{2} \theta}{\partial Z^{2}}\right)$

The fin equation is as follow:

$\frac{\partial \theta}{\partial R}+\frac{\theta}{R}+\frac{1}{R} \frac{\partial \theta}{\partial \emptyset}+\frac{\partial \theta}{\partial Z}=0$

\subsection{Dimensionless Hydraulic Boundary Conditions}

The boundary conditions are given as:

$$
\begin{array}{ll}
\text { at } R=R_{1}, 1 & \frac{1}{R} \frac{\partial\left(R \psi_{r}\right)}{\partial R}=\psi_{\emptyset}=\psi_{z}=0 \\
\text { at } \emptyset=0, \pi & \psi_{r}=\frac{\partial \psi_{\emptyset}}{\partial \emptyset}=\psi_{z}=0 \\
\text { at } Z=0, L & \psi_{r}=\psi_{\varnothing}=\frac{\partial \psi_{z}}{\partial Z}=0
\end{array}
$$

The boundary condition of the fin is:

$\frac{1}{R} \frac{\partial\left(R \psi_{r}\right)}{\partial R}=\frac{\partial \psi_{\emptyset}}{\partial \emptyset}=\frac{\partial \psi_{z}}{\partial Z}=0$

On the fin faces which were located on the following planes (fin base)

At $\mathrm{R}=R_{l} \quad$ for $\quad \phi=0, \pi$

See Fig.2

(fin tip)

At $r=r_{1}+H_{f} \quad$ for $\quad \phi=0, \pi$ 


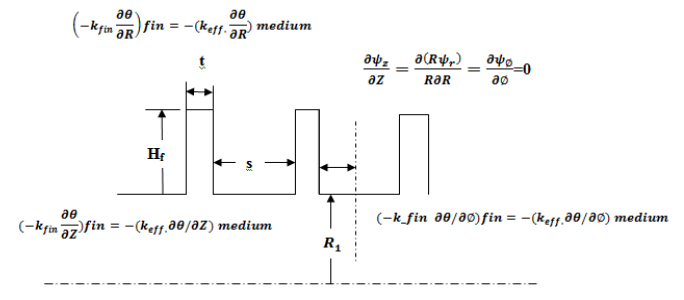

Fig. 2 Fin boundary conditions

\subsection{Dimensionless Thermal Boundary Conditions}

For the temperature field, the dimensionless thermal boundary conditions are:

$\begin{array}{ll}\text { at } R=R_{1}=\frac{r_{1}}{r_{2}} & \theta=1 \\ \text { at } R=R_{2}=1 & \theta=0 \\ \text { at } \emptyset=0, \pi & \frac{\partial \theta}{\partial \emptyset}=0 \\ \text { at } Z=0, L & \frac{\partial \theta}{\partial Z}=0 \\ \text { at } R=H_{1}-\left.k_{\text {fin }} \frac{\partial \theta}{\partial R}\right|_{\text {fin }}=-\left.k_{\text {eff }} \frac{\partial \theta}{\partial R}\right|_{\text {medium }}\end{array}$

at $S_{1}$ for any $R$ and $\emptyset$ and

at $S_{2}$ for any $R$ and $\varnothing$

$-\left.k_{f i n} \frac{\partial \theta}{\partial Z}\right|_{f i n}=-\left.k_{\text {eff }} \frac{\partial \theta}{\partial Z}\right|_{\text {medium }}$

at $\emptyset=0, \pi$ and any $R$

$-\left.k_{f i n} \frac{\partial \theta}{\partial \emptyset}\right|_{\text {fin }}=-\left.k_{\text {eff }} \frac{\partial \theta}{\partial \emptyset}\right|_{\text {medium }}$

$k_{\text {eff }}=(1-\varepsilon) k_{s}+\varepsilon k_{f}$

Fiber orientation can also influence the heat conduction. When fiber axis is perpendicular to heat flux, heat conduction would get the minimum value and conductive conductivity calculated by series model would be the lowest. When fiber axis is parallel to heat flux, conductive conductivity calculated by parallel model would be the largest.

Graphite fibers and epoxy have conductive heat transfer coefficients of 14.74 and $0.19 \mathrm{~W} / \mathrm{m} \mathrm{K}$, respectively. The conductive coefficients of the graphite fibers and the epoxy matrix differ greatly. Consequently, the thermal conductivity of this composite laminate parallel to the fibers is much greater than that perpendicular to the fibers. Graphite/epoxy composite material has a thermal conductivity in parallel direction of fibers of $11.1 \mathrm{~W} / \mathrm{m} \mathrm{K}$ and in perpendicular direction of fibers of $0.87 \mathrm{~W} / \mathrm{m} \mathrm{K}$ and with volumetric percentage of fibers of 75 [13].

The number of grid points used was (21, 31 and 301) grid points in the $\mathrm{R}$ - direction, $\phi$ - direction and and in the $\mathrm{Z}$ direction respectively.

Convergence criterion given by inequality:

maximum error $=\left|\frac{\theta^{n+1}-\theta^{n}}{\theta^{n}}\right| \leq 10^{7}$
International Journal of Computer Applications (0975 - 8887)

Volume 125-No.8, September 2015

\subsection{Calculation of Local and Average Nusselt Number}

Local Nusselt number is the dimensionless parameter indicative of the rate of energy convection from a surface and can be obtained as follows [9]:

$N u=\frac{q\left(r_{2}-r_{1}\right)}{k\left(T_{1}-T_{2}\right)}$

The local Nusselt number $N u_{1}$ and $N u_{2}$ on the inner and the outer surfaces are written in the form [9]:

$N u_{\text {Local } 1}=-\left(1-R_{1}\right)\left(\frac{\partial \theta}{\partial R}\right)_{R=R_{1}}$
$N u_{\text {Local 2 }}=-\left(1-R_{1}\right)\left(\frac{\partial \theta}{\partial R}\right)_{R=R_{1}}$

The mean Nusselt number $N u_{i l}$ and $N u_{2}$ on the inner and the outer surfaces respectively are defined as:

$N u_{1}=-\left(1-R_{1}\right) \frac{1}{\pi L} \int_{0}^{\pi} \int_{0}^{L}\left(\frac{\partial \theta}{\partial R}\right)_{R=R_{1}} d Z d \emptyset$
$N u_{2}=-\left(1-R_{1}\right) \frac{1}{\pi L} \int_{0}^{\pi} \int_{0}^{L}\left(\frac{\partial \theta}{\partial R}\right)_{R=1} d Z d \emptyset$

\section{RESULTS AND DISCUSSION}

\subsection{Isotherms and Streamlines Field}

The isothermal lines and streamlines within the enclosure are presented in a contour maps form in the $(\mathrm{R}-\phi)$ plane.

Fig.3 to Fig. 12 show the isotherms and streamlines for the two cases of maximum and minimum thermal conductivity of the Graphite fibers/epoxy and for different values of Ra, radius ratios $\mathrm{Rr}$ and for horizontal and vertical annulus. In Fig. 3 and Fig. 4 the uniformity and symmetry of the isothermal lines for horizontal annulus is clear and it was observed that for $\mathrm{Rr}=0.5$ in Fig. 4 the intensity of the streamlines is less than that for $\mathrm{Rr}=0.2$ and the cold region for $\mathrm{Rr}=0.2$ is larger because the gap is wider.

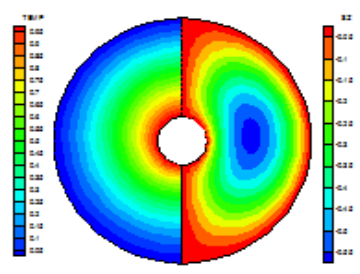

Fig. 3 Isothermal and stream contours for $\operatorname{Rr}=0.2, \mathrm{Ra}^{*}=10$, $\delta=0, \mathrm{k}=11.1 \mathrm{~W} / \mathrm{m} \mathrm{K}$

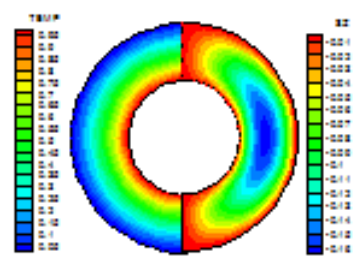

Fig. 4 Isothermal and stream contours for

The streamlines have high intensity at the boundaries and less values in the regions away from the boundary which take beans form and expand as $\delta$ becomes $90^{\circ}$ (vertical annulus) as shown in Fig. 5 and Fig. 6. Increases Ra ${ }^{*}$ for $\delta=0^{\circ}$ results in a thicker cold layer near the bottom wall and a high temperature field near the top wall. More heat is transported upward, and a 
large difference of temperature is observed between the upper and lower parts of the annulus as shown in Fig. 7 and Fig. 8

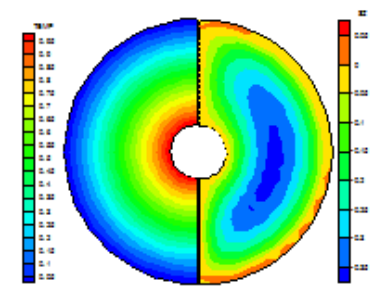

Fig. 5 Isothermal and stream contours for $\operatorname{Rr}=0.2, \mathrm{Ra}^{*}=10$, $\delta=90, \mathrm{k}=11.1 \mathrm{~W} / \mathrm{m} \mathrm{K}$

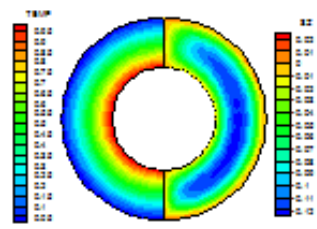

Fig. 6 Isothermal and stream contours for $R r=0.5, R^{*}=10$, $\delta=90, \mathrm{k}=11.1 \mathrm{~W} / \mathrm{m} \mathrm{K}$

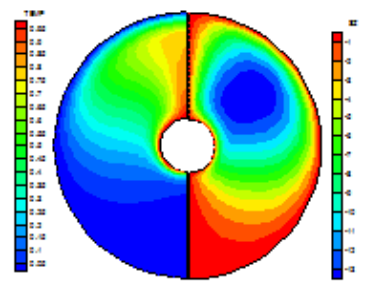

Fig. 7 Isothermal and stream contours for $\mathrm{Rr}=0.2$, $\mathrm{Ra}^{*}=500, \delta=0, k=11.1 \mathrm{~W} / \mathrm{m} \mathrm{K}$

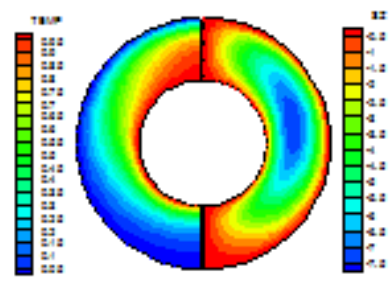

Fig. 8 Isothermal and stream contours for $\operatorname{Rr}=0.5$, $\mathrm{Ra}^{*}=500, \delta=0, k=11.1 \mathrm{~W} / \mathrm{m} \mathrm{K}$

The streamlines have high intensity in the boundaries and at the bottom and as $\delta$ increase to $90^{\circ}$, the center of the streamlines of negative values expand and fade away to the upper region. These figures show clearly that, the isothermal lines closed to the hot and cold cylinders for $\delta=0^{\circ}$, are nearly parallel, indicating that the heat is transferred by conduction for low values of $\mathrm{Ra}^{*}$.

Far away from the inner cylinder, the isothermal lines are deformed from their conductive pattern, and become
International Journal of Computer Applications (0975 - 8887)

Volume 125 - No.8, September 2015

curvilinear indicating that an ascending and descending convective flows occur. These figures can also show the effect of the angles of inclination of the enclosure on the convective heat transfer. The constant temperature lines seem more curvilinear as the angle of inclination increases, and the temperature throughout the enclosure increases which means that the convective flow becomes vigorous. This situation is appeared due to the applying of Darcy's law which shows the effect of the mass density is related to the temperature gradient and the local component of the gravity acceleration vector in the direction of the flow to produce the driving force of the fluid motion inside the enclosure. The fluid near the hot surface gains heat and becomes less dense, whereas the fluid near the cold surface rejects heat and then becomes denser. For vertical annulus the isothermal lines indicate high temperature and so less heat transfer than that for horizontal annulus as shown in Fig. 9 and Fig. 10.

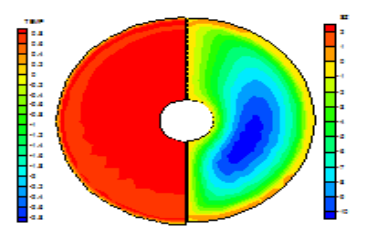

Fig. 9 Isothermal and stream contours for $\mathrm{Rr}=0.2$, $\operatorname{Ra}^{*}=500, \delta=90, k=11.1 \mathrm{~W} / \mathrm{m} \mathrm{K}$

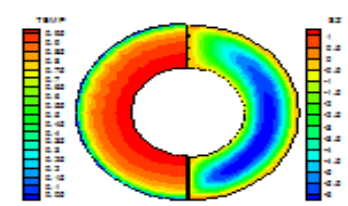

Fig. 10 Isothermal and stream contours for $\mathrm{Rr}=0.5$, $\operatorname{Ra}^{*}=500, \delta=90, k=11.1 \mathrm{~W} / \mathrm{m} \mathrm{K}$

Fig.11 to Fig. 12 show the same behave of isotherms and streamlines for $\mathrm{k}=0.87 \mathrm{~W} / \mathrm{m} \mathrm{K}$ (perpendicular direction of fibers), as in previous figures but of course with less heat transfer, so for $\mathrm{k}=0.87 \mathrm{~W} / \mathrm{m} \mathrm{K}$, the region in the bottom is colder than that for the case of $\mathrm{k}=11.1 \mathrm{~W} / \mathrm{m} \mathrm{K}$ (parallel direction of fibers) taking the same values of $\mathrm{Ra}^{*}, \mathrm{Rr}$ and $\delta$.

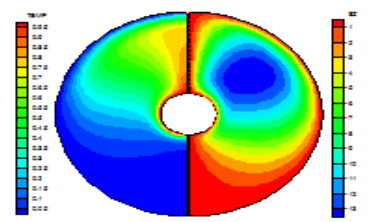

Fig. 11 Isothermal and stream contours for $\mathbf{R r}=0.2$, $\mathrm{Ra}^{*}=500, \delta=0, k=0.87 \mathrm{~W} / \mathrm{m} \mathrm{K}$

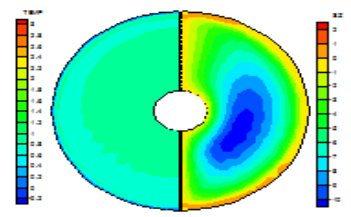

Fig. 12 Isothermal and stream contours for $\mathrm{Rr}=0.2$, $\mathrm{Ra}^{*}=500, \delta=90, \mathrm{k}=0.87 \mathrm{~W} / \mathrm{m} \mathrm{K}$ 


\subsection{Average and Local Nusselt Numbers}

Fig.13 to Fig. 16 show the variation of the average Nusselt number with $\mathrm{Ra}^{*}$ on the outer and inner cylinders and for $\mathrm{k}=0.87 \mathrm{~W} / \mathrm{m} \mathrm{K}$ and $\mathrm{k}=11.1 \mathrm{~W} / \mathrm{m} \mathrm{K}$, respectively for $\delta=0^{\circ}$ and for different values of $\mathrm{Rr}$. It is clear that $\mathrm{Nu}$ increase with the increase of $\mathrm{Ra}^{*}$ and decrease with the decrease of $\mathrm{Rr}$ (larger gap), for the outer cold cylinder.

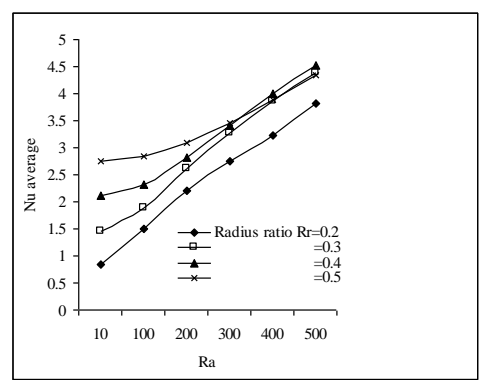

Fig. 13 Variation of $\mathrm{Nu}_{\text {average }}$ with $\mathrm{Ra}$ for $\mathrm{k}=0.87 \mathrm{~W} / \mathrm{m} \mathrm{K}$, $\delta=0$ and for different radius ratios on the outer cylinder

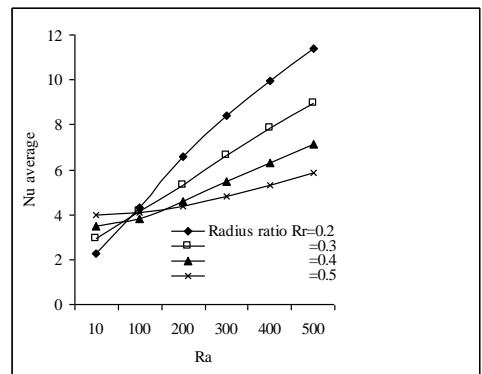

Fig. 14 Variation of $\mathrm{Nu}_{\text {average }}$ with $\mathrm{Ra}$ for $\mathrm{k}=0.87 \mathrm{~W} / \mathrm{m} \mathrm{K}$, $\delta=0$ and different

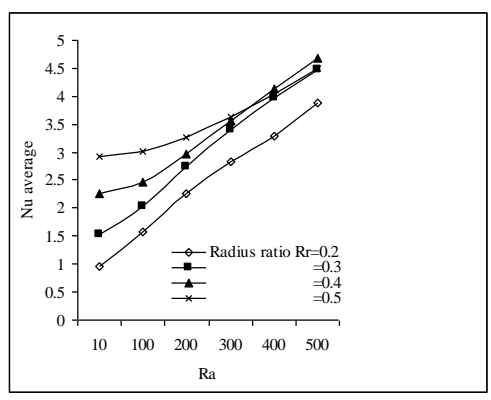

Fig. 15 Variation of $\mathrm{Nu}_{\text {average }}$ with $\mathrm{Ra}$ for $\mathrm{k}=11.1 \mathrm{~W} / \mathrm{m} \mathrm{K}$, $\delta=0$ and for different radius ratios on the outer cylinder

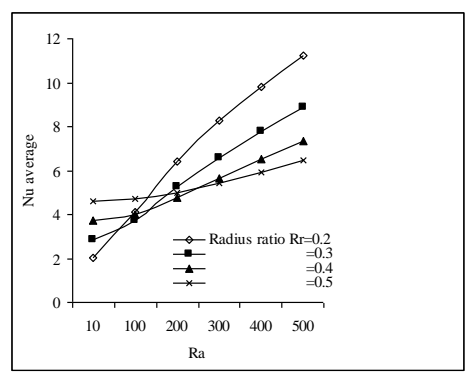

Fig. 16 Variation of $\mathrm{Nu}_{\text {average }}$ with $\mathrm{Ra}$ for $\mathrm{k}=11.1 \mathrm{~W} / \mathrm{m} \mathrm{K}$, $\delta=0$ and for different radius ratios on the inner cylinder

The deviation between the average $\mathrm{Nu}$ in the two cases is clear in Fig.17 and Fig. 18 which is equal to $5.1 \%$ for $\delta=0^{\circ}$ and $10 \%$ for $\delta=90^{\circ}$.

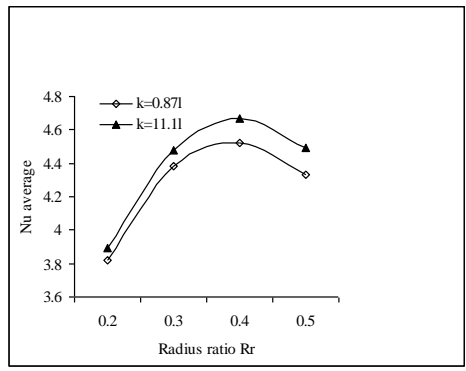

Fig. 17 Variation of $\mathrm{Nu}_{\text {average }}$ with radius ratios For $\mathrm{Ra}=500, \delta=0^{\circ}$ on the outer cylinder

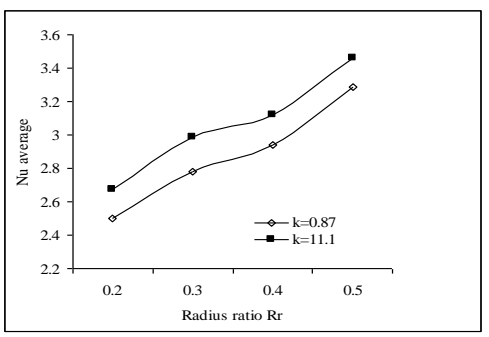

Fig. 18 Variation of $\mathrm{Nu}_{\text {average }}$ with radius ratios For $\mathbf{R a}=500, \delta=90^{\circ}$ on the outer cylinder

Fig.19 to Fig. 22 show the variation of the average Nusselt number with $\mathrm{Ra}^{*}$ on the outer and inner cylinders and for $\mathrm{k}=0.87 \mathrm{~W} / \mathrm{m} \mathrm{K}$ and $\mathrm{k}=11.1 \mathrm{~W} / \mathrm{m} \mathrm{K}$, respectively for $\delta=90^{\circ}$ and for different values of Rr. These figures indicate that the heat transfer is lower than that for $\delta=0^{\circ}$.

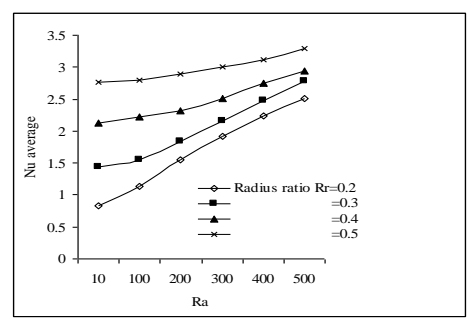

Fig. 19 Variation of $\mathrm{Nu}_{\text {average }}$ with $\mathrm{Ra}$ for $\mathrm{k}=0.87 \mathrm{~W} / \mathrm{m} \mathrm{K}$, $\delta=90$ and for different radius ratios on the outer cylinder

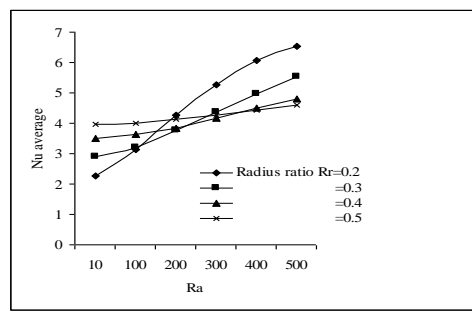

Fig. 20 Variation of $\mathrm{Nu}_{\text {average }}$ with $\mathrm{Ra}$ for $\mathrm{k}=0.87 \mathrm{~W} / \mathrm{m} \mathrm{K}$, $\delta=90$ and different radius ratios on the inner cylinder

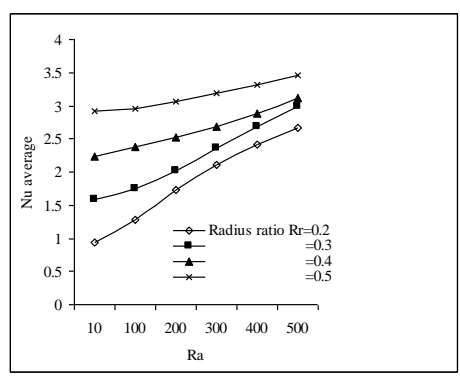

Fig. 21 Variation of $\mathrm{Nu}$ average with Ra fork $=11.1 \mathrm{~W} / \mathrm{m} \mathrm{K}$, $\delta=90$ and for different radius ratios on the outer cylinder 


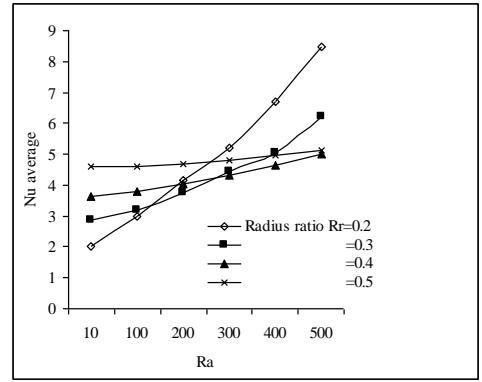

Fig. 22 Variation of $\mathrm{Nu}_{\text {average }}$ with $\mathrm{Ra}$ for $\mathrm{k}=11.1 \mathrm{~W} / \mathrm{m} \mathrm{K}$, $\delta=90$ and different radius ratios on the inner cylinder

The distribution of the local Nusselt number along the dimensionless length of the cylinder is illustrated in Fig. 23 to Fig. 32 for $\delta=0^{\circ}$ and $\delta=90^{\circ}$ respectively.

As shown in Fig. 23 and Fig. 24, the effect of the fins attached to the inner cylinder is very significant for $\delta=0^{\circ}$ and the curve will be wavy and this waviness increase as $\mathrm{Ra}^{*}$ increase as shown in Fig. 24 to Fig. 26.

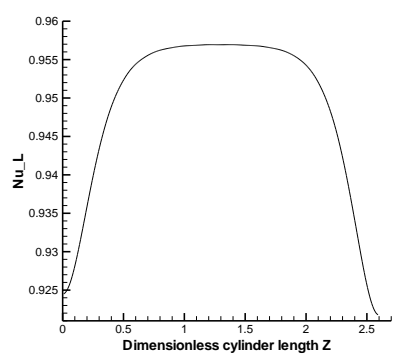

Fig. 23 Variation of $\mathrm{Nu}_{\text {Local }}$ with cylinder length for $\mathrm{Ra}=10, \mathrm{Rr}=0.2, \mathrm{k}=11.1 \mathrm{~W} / \mathrm{m} \mathrm{K} \delta=0^{\circ}$

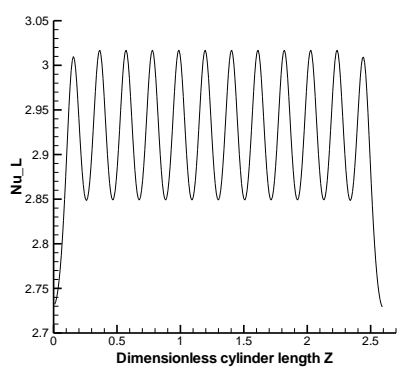

Fig. 24 Variation of $\mathrm{Nu}_{\text {Local }}$ with cylinder length for $\mathrm{Ra}=10, \mathrm{Rr}=0.5$ and $\mathrm{k}=11.1 \mathrm{~W} / \mathrm{m} \mathrm{K} \delta=90^{\circ}$

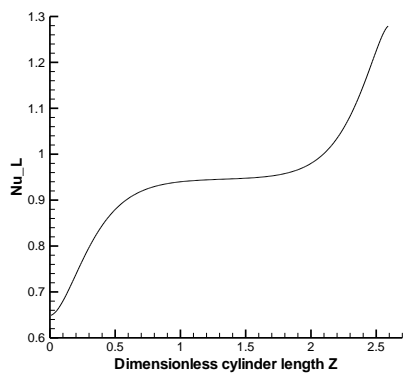

Fig. 25 Variation of $\mathrm{Nu}_{\text {Local }}$ with cylinder length for $\mathrm{Ra}=10, \mathrm{Rr}=0.2$ and $\mathrm{k}=11.1 \mathrm{~W} / \mathrm{m} \mathrm{K} \delta=90^{\circ}$

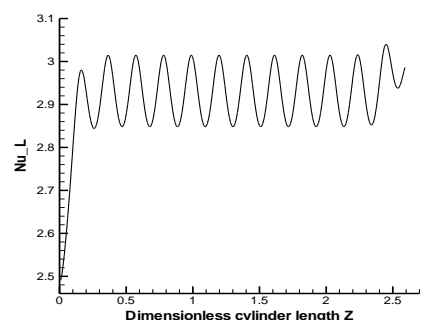

Fig. 26 Variation of $\mathrm{Nu}_{\text {Local }}$ with cylinder length for $\mathrm{Ra}=10, \mathrm{Rr}=0.5$ and $\mathrm{k}=11.1 \mathrm{~W} / \mathrm{m} \mathrm{K}$ and $\delta=90^{\circ}$

For k=0.87 W/m K, in Fig. 27 to Fig. 38 the local Nusselt number was less but with the same behave as the previous figures for $\mathrm{k}=11.1 \mathrm{~W} / \mathrm{m} \mathrm{K}$.

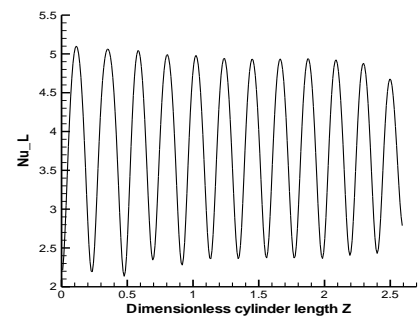

Fig. 27 Variation of $\mathrm{Nu}_{\text {Local }}$ with cylinder length for $\mathrm{Ra}=500, \mathrm{Rr}=0.2, \mathrm{k}=11.1 \mathrm{~W} / \mathrm{m} \mathrm{K}$ and $\delta=0^{\circ}$

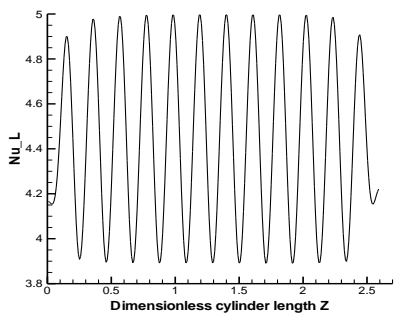

Fig. 28 Variation of $\mathrm{Nu}_{\text {Local }}$ with cylinder length for $\mathrm{Ra}=500, \mathrm{Rr}=0.5$ and $\mathrm{k}=11.1 \mathrm{~W} / \mathrm{m} \mathrm{K} \delta=0^{\circ}$

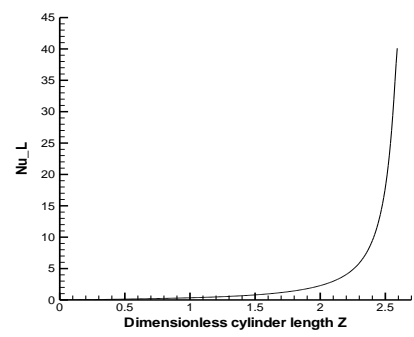

Fig. 29 Variation of $\mathrm{Nu}_{\text {Local }}$ with cylinder length for

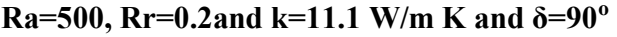

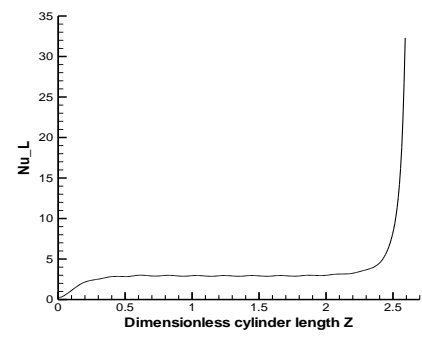

Fig. 30 Variation of $\mathrm{Nu}_{\text {Local }}$ with cylinder length for $\mathrm{Ra}=500, \mathrm{Rr}=0.5$ and $\mathrm{k}=11.1$ and $\mathrm{W} / \mathrm{m} \mathrm{K} \delta=90^{\circ}$ 


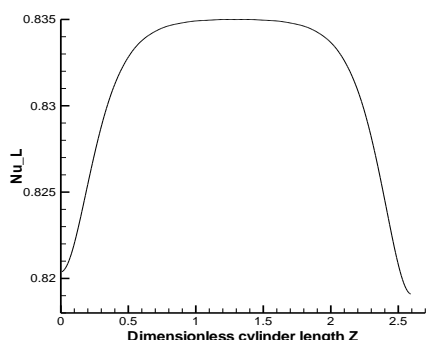

Fig. 31 Variation of $\mathrm{Nu}_{\text {Local }}$ with cylinder length for $\mathrm{Ra}=10, \mathrm{Rr}=0.2, \mathrm{k}=0.87 \mathrm{~W} / \mathrm{m} \mathrm{K}$ and $\delta=0^{\circ}$

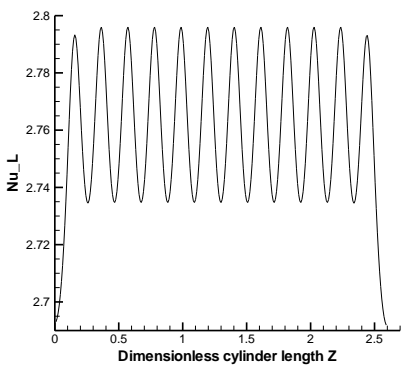

Fig. 32Variation of $\mathrm{Nu}_{\mathrm{Local}}$ with cylinder length for $\mathrm{Ra}=\mathbf{1 0}$, $\mathrm{Rr}=0.5, \mathrm{k}=0.87 \mathrm{~W} / \mathrm{m} \mathrm{K}$ and $\delta=0^{\circ}$

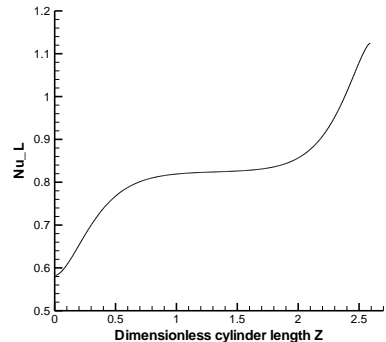

Fig. 33Variation of $\mathrm{Nu}_{\text {Local }}$ with cylinder length for $\mathrm{Ra}=10, \mathrm{Rr}=0.2, \mathrm{k}=0.87 \mathrm{~W} / \mathrm{m} \mathrm{K}$ and $\delta=90^{\circ}$

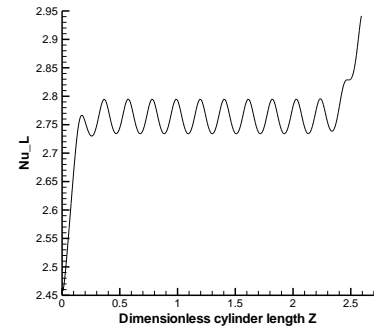

Fig. 34Variation of $\mathrm{Nu}_{\text {Local }}$ with cylinder length for $\mathrm{Ra}=\mathbf{1 0}$, $\mathrm{Rr}=0.5, \mathrm{k}=0.87 \mathrm{~W} / \mathrm{m} \mathrm{K}$ and $\delta=90^{\circ}$

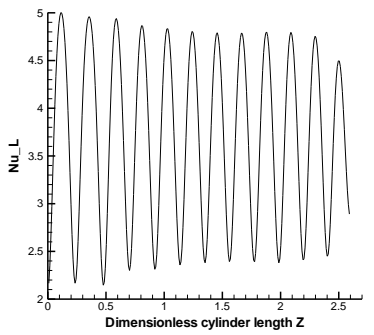

Fig. 35Variation of $\mathrm{Nu}_{\text {Local }}$ with cylinder length for $\mathrm{Ra}=500, \mathrm{Rr}=0.2, \mathrm{k}=0.87 \mathrm{~W} / \mathrm{m} \mathrm{K}$ and $\delta=0^{\circ}$

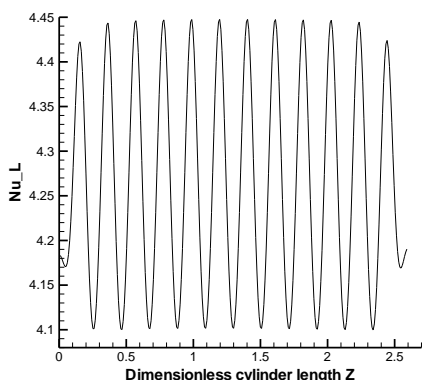

Fig. 36Variation of $\mathrm{Nu}_{\mathrm{Local}}$ with cylinder length for $R a=500, R r=0.5, k=0.87 \mathrm{~W} / \mathrm{m} \mathrm{K}$ and $\delta=0^{\circ}$

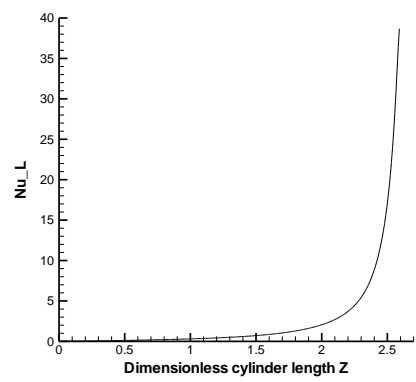

Fig. 37Variation of $\mathrm{Nu}_{\text {Local }}$ with cylinder length for $\mathrm{Ra}=500, \mathrm{Rr}=0.2, \mathrm{k}=0.87 \mathrm{~W} / \mathrm{m} \mathrm{K}$ and $\delta=90^{\circ}$

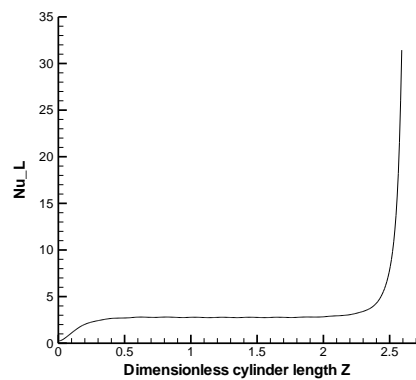

Fig. 38Variation of $\mathrm{Nu}_{\text {Local }}$ with cylinder length for $\mathrm{Ra}=500, \mathrm{Rr}=0.5 \mathrm{k}=0.87 \mathrm{~W} / \mathrm{m} \mathrm{K} \delta=90^{\circ}$

Correlations were deduced from the numerical data which are given as:

For $\mathrm{k}=11.1 \mathrm{~W} / \mathrm{m} \mathrm{K}$ :

$N u_{\text {out }}=\frac{1.923 R a^{0.176} R r^{0.5667}}{\delta^{0.011}}$

For k=0.87 W/m K:

$N u_{\text {out }}=\frac{1.67 R a^{0.185} R r^{0.59}}{\delta^{0.013}}$

\section{CONCLUSIONS}

The following major conclusions can be obtained:

1- Nusselt number decrease with the decrease of the radius ratio $\mathrm{Rr}$ (which means larger gap) for the outer cold cylinder.

2- The deviation between the average $\mathrm{Nu}$ for the maximization and minimization of the thermal conductivity is equal to $5.1 \%$ for horizontal annulus $\delta=0^{\circ}$ and $10 \%$ for vertical annulus $\delta=90^{\circ}$. 
3- For all parameters, results showed that the average $\mathrm{Nu}$ number increases with an increase in modified Rayleigh number and decrease with the increase of $\delta$ for high values of $\mathrm{Ra}^{*}$, but hardly affected by $\delta$ for low values of $\mathrm{Ra}^{*}$.

4- Local $\mathrm{Nu}$ increases with the length of the cylinder and the effect of the fins attached to the inner cylinder is more significant for the horizontal cylinder because of its hindering effect.

5- Extension of the present work is recommended for a future work to take nanofluid as the working fluid and take other configurations to investigate the enhancement in the heat transfer and use many types of nanoparticles for the purpose of comparison.

\section{REFERENCES}

[1] Bo Yuan, Shuqiang Ding, Dongdong Wang, Gang Wang, Hongxia Li, 2012, Heat insulation properties of silica aerogel/glass fiber composites fabricated by press forming, Materials Letters 75, 204-206.

[2] Hubert Jopek and Tomasz Strek, 2011, Optimization of the effective thermal conductivity of a composite, Convection and Conduction Heat Transfer, Poznan University of Technology, Institute Of Applied Mechanics Poland, 17, October, 197-214.

[3] Norouzi M., Amiri Delouei A., Seilsepour M., 2013, A general exact solution for heat conduction in multilayer spherical composite laminates, Composite Structures $106,288-295$.

[4] Sangwook Sihn, Sabyasachi Ganguli, David P. Anderson, Ajit K. Roy, 2012, Enhancement of throughthickness thermal conductivity of sandwich construction using carbon foam, Composites Science and Technology 72, 767-773.

[5] Dun-Yen Kang, Christopher W. Jones, Sankar Nair, 2011, Modeling molecular transport in composite membranes with tubular fillers, Journal of Membrane Science $381,50-63$

[6] Wang H.M., Liu C.B., 2013, Analytical solution of twodimensional transient heat conduction in fiber-reinforced cylindrical composites, International Journal of Thermal Sciences 69, 43-52.

[7] Gaosheng Wei, Yusong Liu, Xinxin Zhang, Xiaoze Du, 2013, Radiative heat transfer study on silica aerogel and its composite insulation materials, Journal of NonCrystalline Solids 362, 231-236.

[8] Nield D. A. and Bejan A., "Convection in Porous Media”, Springer-Verlag, New York, 1999.

[9] Fukuda K., Takata Y., Hasegawa S., Shimomura H. and Sanokawa K., 1980, Three - Dimensional Natural Convection in a Porous Medium Between Concentric Inclined Cylinders, Proc. $19^{\text {th }}$ Natl Heat Transfer Conf., Vol. HTD -8, 97 - 103

[10] Kayhani M.H., Norouzi M., Amiri Delouei A., 2012, A general analytical solution for heat conduction in cylindrical multilayer composite laminates, International Journal of Thermal Sciences 52, 73- 82. 